\title{
COINCIDENCE AND FIXED POINT THEOREMS FOR FUNCTIONS IN $S$-KKM CLASS ON GENERALIZED CONVEX SPACES
}

\author{
TIAN-YUAN KUO, YOUNG-YE HUANG, JYH-CHUNG JENG, AND \\ CHEN-YUH SHIH
}

Received 25 October 2004; Revised 13 July 2005; Accepted 1 September 2005

We establish a coincidence theorem in $S$-KKM class by means of the basic defining property for multifunctions in S-KKM. Based on this coincidence theorem, we deduce some useful corollaries and investigate the fixed point problem on uniform spaces.

Copyright (C) 2006 Tian-Yuan Kuo et al. This is an open access article distributed under the Creative Commons Attribution License, which permits unrestricted use, distribution, and reproduction in any medium, provided the original work is properly cited.

\section{Introduction}

A multimap $T: X \rightarrow 2^{Y}$ is a function from a set $X$ into the power set $2^{Y}$ of $Y$. If $H, T$ : $X \rightarrow 2^{Y}$, then the coincidence problem for $H$ and $T$ is concerned with conditions which guarantee that $H(\hat{x}) \cap T(\hat{x}) \neq \varnothing$ for some $\hat{x} \in X$. Park [11] established a very general coincidence theorem in the class $\mathbf{U}_{c}^{k}$ of admissible functions, which extends and improves many results of Browder [1, 2], Granas and Liu [6].

On the other hand, Huang together with Chang et al. [3] introduced the S-KKM class which is much larger than the class $\mathbf{U}_{c}^{k}$. A lot of interesting and generalized results about fixed point theory on locally convex topological vector spaces have been studied in the setting of S-KKM class in [3]. In this paper, we will at first construct a coincidence theorem in $S$-KKM class on generalized convex spaces by means of the basic defining property for multimaps in S-KKM class. And then based on this coincidence theorem, we deduce some useful corollaries and investigate the fixed point problem on uniform spaces.

\section{Preliminaries}

Throughout this paper, $\langle Y\rangle$ denotes the class of all nonempty finite subsets of a nonempty set $Y$. The notation $T: X \multimap Y$ stands for a multimap from a set $X$ into $2^{Y} \backslash\{\varnothing\}$. For a multimap $T: X \rightarrow 2^{Y}$, the following notations are used:
(a) $T(A)=\bigcup_{x \in A} T(x)$ for $A \subseteq X$;
(b) $T^{-}(y)=\{x \in X: y \in T(x)\}$ for $y \in Y$;
(c) $T^{-}(B)=\{x \in X: T(x) \cap B \neq \varnothing\}$ for $B \subseteq Y$.

Hindawi Publishing Corporation

Fixed Point Theory and Applications

Volume 2006, Article ID 72184, Pages 1-9

DOI 10.1155/FPTA/2006/72184 
All topological spaces are supposed to be Hausdorff. Let $X$ and $Y$ be two topological spaces. A multimap $T: X \rightarrow 2^{Y}$ is said to be

(a) upper semicontinuous (u.s.c.) if $T^{-}(B)$ is closed in $X$ for each closed subset $B$ of $Y$;

(b) compact if $T(X)$ is contained in a compact subset of $Y$;

(c) closed if its graph $\operatorname{Gr}(T)=\{(x, y): y \in T(x), x \in X\}$ is a closed subset of $X \times Y$.

Lemma 2.1 (Lassonde [9, Lemma 1]). Let $X$ and $Y$ be two topological spaces and $T: X \multimap$ $Y$.

(a) If $Y$ is regular and $T$ is u.s.c. with closed values, then $T$ is closed. Conversely, if $Y$ is compact and $T$ is closed, then $T$ is u.s.c. with closed values.

(b) If $T$ is u.s.c. and compact-valued, then $T(A)$ is compact for any compact subset $A$ of $X$.

Let $X$ be a subset of a vector space and $D$ a nonempty subset of $X$. Then $(X, D)$ is called a convex space if the convex hull $\operatorname{co}(A)$ of any $A \in\langle D\rangle$ is contained in $X$ and $X$ has a topology that induces the Euclidean topology on such convex hulls. A subset $C$ of $(X, D)$ is said to be $D$-convex if $\operatorname{co}(A) \subseteq C$ for any $A \in\langle D\rangle$ with $A \subseteq C$. If $X=D$, then $X=(X, X)$ becomes a convex space in the sense of Lassonde [9]. The concept of convexity is further generalized under an extra condition by Park and Kim [12]. Later, Lin and Park [10] give the following definition by removing the extra condition.

Definition 2.2. A generalized convex space or a $G$-convex space $(X, D ; \Gamma)$ consists of a topological space $X$, a nonempty subset $D$ of $X$ and a map $\Gamma:\langle D\rangle \multimap X$ such that for each $A \in\langle D\rangle$ with $|A|=n+1$, there exists a continuous function $\varphi_{A}: \Delta_{n} \rightarrow \Gamma(A)$ such that $J \in\langle A\rangle$ implies $\varphi_{A}\left(\Delta_{J}\right) \subseteq \Gamma(J)$, where $\Delta_{J}$ denotes the face of $\Delta_{n}$ corresponding to $J \in\langle A\rangle$.

A subset $K$ of a $G$-convex space $(X, D ; \Gamma)$ is said to be $\Gamma$-convex if for any $A \in\langle K \cap D\rangle$, $\Gamma(A) \subseteq K$.

In what follows we will express $\Gamma(A)$ by $\Gamma_{A}$, and we just say that $(X, \Gamma)$ is a $G$-convex space provided that $D=X$.

The $c$-space introduced by Horvath [7] is an example of $G$-convex space.

For topological spaces $X$ and $Y, \mathscr{C}(X, Y)$ denote the class of all continuous (singlevalued) functions from $X$ to $Y$.

Given a class $\mathscr{L}$ of multimaps, $\mathscr{L}(X, Y)$ denotes the set of multimaps $T: X \rightarrow 2^{Y}$ belonging to $\mathscr{L}$, and $\mathscr{L}_{c}$ the set of finite composites of multimaps in $\mathscr{L}$. Park and Kim [12] introduced the class $\mathbf{U}$ to be the one satisfying

(a) U contains the class $\mathscr{C}$ of (single-valued) continuous functions;

(b) each $T \in \mathbf{U}_{c}$ is upper semicontinuous and compact-valued; and

(c) for any polytope $P$, each $T \in \mathbf{U}_{c}(P, P)$ has a fixed point.

Further, Park defined the following

$$
\begin{aligned}
T \in \mathbf{U}_{c}^{k}(X, Y) \Longleftrightarrow & \text { for any compact subset } K \text { of } X, \text { there is a } \\
& \Gamma \in \mathbf{U}_{c}(X, Y) \text { such that } \Gamma(x) \subseteq T(x) \text { for each } x \in K .
\end{aligned}
$$


A uniformity for a set $X$ is a nonempty family $\mathcal{U}$ of subsets of $X \times X$ such that

(a) each member of $\mathcal{U}$ contains the diagonal $\Delta$;

(b) if $U \in \mathcal{U}$, then $U^{-1} \in \mathcal{U}$;

(c) if $U \in \mathcal{U}$, then $V \circ V \subseteq U$ for some $V$ in $U$;

(d) if $U$ and $V$ are members of $\mathcal{U}$, then $U \cap V \in \mathcal{U}$; and

(e) if $U \in \mathcal{U}$ and $U \subseteq V \subseteq X \times X$, then $V \in \mathcal{U}$.

If $(X, \mathcal{U})$ is a uniform space the topology $\mathscr{T}$ induced by $\mathcal{U}$ is the family of all subsets $W$ of $X$ such that for each $x$ in $W$ there is $U$ in $U$ such that $U[x] \subseteq W$, where $U[x]$ is defined as $\{y \in X:(x, y) \in U\}$. For details of uniform spaces we refer to [8].

\section{The results}

The concept of $S$-KKM property of [3] can be extented to G-convex spaces.

Definition 3.1. Let $X$ be a nonempty set, $(Y, D ; \Gamma)$ a $G$-convex space and $Z$ a topological space. If $S: X \multimap D, T: Y \multimap Z$ and $F: X \multimap Z$ are three multimaps satisfying

$$
T\left(\Gamma_{S(A)}\right) \subseteq F(A)
$$

for any $A \in\langle X\rangle$, then $F$ is called a $S$-KKM mapping with respect to $T$. If the multimap $T$ : $Y \multimap Z$ satisfies that for any $S$-KKM mapping $F$ with respect to $T$, the family $\{\overline{F(x)}: x \in$ $X\}$ has the finite intersection property, then $T$ is said to have the $S$-KKM property. The class $S$ - $\operatorname{KKM}(X, Y, Z)$ is defined to be the set $\{T: X \multimap Y: T$ has the $S$-KKM property $\}$.

When $D=Y$ is a nonempty convex subset of a linear space with $\Gamma_{B}=\operatorname{co}(B)$ for $B \in$ $\langle Y\rangle$, the $S-\operatorname{KKM}(X, Y, Z)$ is just that as in [3]. In the case that $X=D$ and $S$ is the identity mapping $1_{D}, S$ - $\operatorname{KKM}(X, Y, Z)$ is abbreviated as $\operatorname{KKM}(Y, Z)$, and a $1_{D}$-KKM mapping with respect to $T$ is called a KKM mapping with respect to $T$, and $1_{D}$-KKM property is called KKM property. Just as [3, Propositions 2.2 and 2.3], for $X$ a nonempty set, $(Y, D ; \Gamma)$ a $G$-convex space, $Z$ a topological space and any $S \multimap D$, one has $T \in \operatorname{KKM}(Y, Z) \subseteq S$ $\operatorname{KKM}(X, Y, Z)$. By the corollary to [13, Theorem 2], we have $\mathbf{U}_{c}^{k}(Y, Z) \subseteq \operatorname{KKM}(Y, Z)$, and so $\mathbf{U}_{c}^{k}(Y, Z) \subseteq S-\operatorname{KKM}(X, Y, Z)$.

Here we like to give a concrete multimap $T$ having KKM property on a $G$-convex space. Let $X=[0,1] \times[0,1]$ be endowed with the Euclidean metric. For any $A=\left\{\mathbf{x}_{1}, \ldots, \mathbf{x}_{n}\right\} \in$ $\langle\mathrm{X}\rangle$, define $\Gamma_{A}=\bigcup_{i=1}^{n}\left[\mathbf{0}, \mathbf{x}_{i}\right]$, where $\left[\mathbf{0}, \mathbf{x}_{i}\right]$ denotes the line segment joining $\mathbf{0}$ and $\mathbf{x}_{i} . \mathrm{It}$ is easy to see that $(X, \Gamma)$ is a $c$-space, and so it is a $G$-convex space. Let $T: X \multimap X$ be defined by $T(\mathbf{x})=[(0,0),(0,1)] \cup[(0,0),(1,0)]$. If $F: X \multimap X$ is any KKM mapping with respect to $T$, then for any $A=\left\{\mathbf{x}_{1}, \ldots, \mathbf{x}_{n}\right\} \in\langle\mathrm{X}\rangle$, since $T\left(\Gamma_{A}\right) \subseteq F(A)$ and $(0,0) \in T(0,0)$, we infer that $(0,0) \in T\left(\mathbf{x}_{i}\right) \subseteq F\left(\mathbf{x}_{i}\right)$ for any $i=1, \ldots, n$, so $(0,0) \in \bigcap_{i=1}^{n} F\left(\mathbf{x}_{i}\right)$. This shows that $T$ has the KKM property.

A subset $B$ of a topological space $Z$ is said to be compactly open if for any compact subset $K$ of $Z, K \cap B$ is open in $K$. We begin with the following coincidence theorem.

Theorem 3.2. Let $X$ be any nonempty set, $(Y, D ; \Gamma)$ a $G$-convex space and $Z$ a topological space. Suppose $s: X \rightarrow D, W: D \rightarrow 2^{Z}, H: Y \rightarrow 2^{Z}$ and $T \in s-\operatorname{KKM}(X, Y, Z)$ satisfy the 
4 Coincidence and fixed point theorems in S-KKM class

following conditions:

(3.2.1) $T$ is compact;

(3.2.2) for any $y \in D, W(y) \subseteq H(y)$ and $W(y)$ is compactly open in $Z$;

(3.2.3) for any $z \in T(Y), M \in\left\langle W^{-}(z)\right\rangle$ implies that $\Gamma_{M} \subseteq H^{-}(z)$;

(3.2.4) $\overline{T(Y)} \subseteq \bigcup_{x \in X} W(s(x))$.

Then $T$ and $H$ have a coincidence point.

Proof. We prove the theorem by contradiction. Assume that $T(y) \cap H(y)=\varnothing$ for any $y \in Y$. Put $K=\overline{T(Y)}$. By (3.2.1), $K$ is a compact subset of $Z$. Define $F: X \rightarrow 2^{Z}$ by

$$
F(x)=K \backslash W(s(x))
$$

for $x \in X$. Since $W(s(x))$ is compactly open, $F(x)$ is closed for each $x \in X$. The assumption that $T(y) \cap H(y)=\varnothing$ for any $y \in Y$ implies that $T(s(x)) \cap H(s(x))=\varnothing$ for any $x \in X$, so

$$
\begin{aligned}
\varnothing \neq T(s(x)) & \subseteq K \backslash H(s(x)) \\
& \subseteq K \backslash W(s(x)) \\
& =F(x) .
\end{aligned}
$$

Hence $F$ is a nonempty and compact-valued multimap. Since

$$
\begin{aligned}
\bigcap_{x \in X} F(x) & =\bigcap_{x \in X}(K \backslash W(s(x))) \\
& =K \backslash \bigcup_{x \in X} W(s(x)) \\
& \subseteq K \backslash K \quad \text { by }(3.2 .4) \\
& =\varnothing,
\end{aligned}
$$

$F$ is not a $s$-KKM mapping with respect to $T$. Hence there is $A=\left\{x_{1}, \ldots, x_{n}\right\} \in\langle X\rangle$ such that

$$
T\left(\Gamma_{\left\{s\left(x_{1}\right), \ldots, s\left(x_{n}\right)\right\}}\right) \nsubseteq \bigcup_{i=1}^{n} F\left(x_{i}\right) .
$$

Choose $\hat{y} \in \Gamma_{\left\{s\left(x_{1}\right), \ldots, s\left(x_{n}\right)\right\}}$ and $\hat{z} \in T(\hat{y})$ such that $\hat{z} \notin \bigcup_{i=1}^{n} F\left(x_{i}\right)$. It follows from

$$
\begin{aligned}
\hat{z} & \in K \backslash \bigcup_{i=1}^{n} F\left(x_{i}\right) \\
& =\bigcap_{i=1}^{n}\left(K \backslash F\left(x_{i}\right)\right) \\
& \subseteq \bigcap_{i=1}^{n} W\left(s\left(x_{i}\right)\right) \\
& \subseteq \bigcap_{i=1}^{n} H\left(s\left(x_{i}\right)\right)
\end{aligned}
$$


that $s\left(x_{i}\right) \in W^{-}(\hat{z}) \subseteq H^{-}(\hat{z})$ for any $i \in\{1, \ldots, n\}$. Therefore by $(3.2 .3), \Gamma_{\left\{s\left(x_{1}\right), \ldots, s\left(x_{n}\right)\right\} \subseteq}$ $H^{-}(\hat{z})$. In particular, $\hat{y} \in H^{-}(\hat{z})$, and so $\hat{z} \in H(\hat{y}) \cap T(\hat{y})$, a contradiction. This completes the proof.

Corollary 3.3. Let $(Y, D)$ be a convex space and $Z$ a topological space. Suppose $H: Y \rightarrow 2^{Z}$ and $T \in \operatorname{KKM}(Y, Z)$ satisfy the following conditions:

(3.3.1) $T$ is compact;

(3.3.2) for any $z \in T(Y), H^{-}(z)$ is D-convex;

(3.3.3) $\overline{T(Y)} \subseteq \bigcup_{y \in D} \operatorname{Int}(H(y))$.

Then $T$ and $H$ have a coincidence point.

Proof. Putting $X=D, s: X \rightarrow D$ be the identity mapping $1_{D}$ and $W: D \rightarrow 2^{Z}$ be defined by $W(y)=\operatorname{Int}(H(y))$ in the above theorem, the result follows immediately.

Here we like to mention that Corollary 3.3 is an improvement for Theorem 4 of Chang and Yen [4], where except the conditions (3.3.1) (3.3.3), they require $T$ be closed. For $\mathbf{U}_{c}^{k}(Y, Z)$ instead of $\operatorname{KKM}(Y, Z)$, Corollary 3.3 is due to Park [11]. We now give a concrete example showing that Corollary 3.3 extends both of [4, Theorem 4] and [11, Theorem 2] properly. Let $X=[0,1]$ and $V$ be any convex open subset of 0 in $\mathbb{R}$. Define $T: X \multimap X$ by $T(x)=\{1\}$ for $x \in[0,1)$; and $[0,1)$ for $x=1$, and $H: X \multimap X$ by $H(x)=(x+V) \cap X$. Then we have

(a) $T$ belongs to $\operatorname{KKM}(X, X)$ and is compact;

(b) $H^{-}(y)$ is convex for each $y \in X$, and

(c) each $H(x)$ is open and $\overline{T(X)} \subseteq \bigcup_{x \in X} H(x)$.

Thus, Corollary 3.3 guarantees that $T(\hat{x}) \cap H(\hat{x}) \neq \varnothing$ for some $\hat{x} \in[0,1]$. But, Theorem 4 of Chang and Yen [4] is not applicable in this case because $T$ is not closed. On the other hand, if $T \in \mathbf{U}_{c}^{k}(X, X)$, then there would exist $\Gamma \in \mathbf{U}_{c}(X, X)$ such that $\Gamma(x) \subseteq T(x)$ for each $x \in[0,1]$. Since $X$ is a polytope, $\Gamma$ must have a fixed a point which is impossible by noting that $T$ has no fixed point. Consequently, $T \notin \mathbf{U}_{c}^{k}(X, X)$, and hence we can not apply Theorem 2 of Park [11] to conclude that $T$ and $H$ have a coincidence point.

Corollary 3.4. Let $X$ be any nonempty set, $(Y, D)$ a convex space and $Z$ a topological space. Suppose $s: X \rightarrow D, H: Y \rightarrow 2^{Z}$ and $T \in s-\operatorname{KKM}(X, Y, Z)$ satisfy the following conditions:

(3.4.1) $T$ is compact;

(3.4.2) for any $z \in T(Y), H^{-}(z)$ is $D$-convex;

(3.4.3) $\overline{T(Y)} \subseteq \bigcup_{x \in X} \operatorname{Int}(H(s(x)))$.

Then $T$ and $H$ have a coincidence point.

Proof. In Theorem 3.2, putting $W: D \rightarrow 2^{Z}$ be $W(y)=\operatorname{Int}(H(y))$ for each $y \in Y$, the result follows immediately.

LEMma 3.5 (Lassonde [9, Lemma 2]). Let $Y$ be a nonempty subset of a topological vector space $E, T: Y \rightarrow 2^{E}$ a compact and closed multimap and $i: Y \rightarrow E$ the inclusion map. Then for each closed subset $B$ of $Y,(T-i)(B)$ is closed in $E$.

Corollary 3.6. Let $X$ be any nonempty set and $Y, C$ be two nonempty convex subsets of a locally convex topological vector space $E$. Suppose $s: X \rightarrow Y$ and $T \in s-\operatorname{KKM}(X, Y, Y+C)$ satisfy the following conditions (3.6.1), (3.6.2) and any one of (3.6.3), (3.6.3)' and (3.6.3)'". 
6 Coincidence and fixed point theorems in S-KKM class

(3.6.1) $T$ is compact and closed.

(3.6.2) $\overline{T(Y)} \subseteq s(X)+C$.

(3.6.3) $Y$ is closed and $C$ is compact.

(3.6.3)' $Y$ is compact and $C$ is closed.

$(3.6 .3)^{\prime \prime} C=\{0\}$.

Then there is $\hat{y} \in Y$ such $(\hat{y}+C) \cap T(\hat{y}) \neq \varnothing$.

Proof. Let $V$ be any convex open neighborhood of $0 \in E$ and $K=\overline{T(Y)}$. Define $H: Y \rightarrow$ $2^{Y+C}$ to be $H(y)=(y+C+V) \cap K$ for each $y \in Y$. Each $H(y)$ is open in $K$ and $H^{-}(z)=$ $(z-C-V) \cap Y$ is convex for any $z \in K$. Moreover,

$$
\begin{aligned}
\bigcup_{x \in X} H(s(x)) & =\bigcup_{x \in X}((s(x)+C+V) \cap K) \\
& =(s(X)+C+V) \cap K \\
& =\overline{T(Y)} \quad \text { by (3.6.2). }
\end{aligned}
$$

Therefore, it follows from Corollary 3.4 that there are $y_{V} \in Y$ and $z_{V} \in K$ such that $z_{V} \in$ $T\left(y_{V}\right) \cap H\left(y_{V}\right)$. Then in view of the definition of $H, z_{V}-y_{V} \in C+V$. Up to now, we have proved the assertion.

(*) For each convex open neighborhood $V$ of 0 in $E,(T-i)(Y) \cap(C+V) \neq \varnothing$, where $i: Y \rightarrow E$ is the inclusion map.

Now take into account of conditions (3.6.3), (3.6.3) $)^{\prime}$ and (3.6.3)". Suppose (3.6.3) holds. Since $Y$ is closed, so is $(T-i)(Y)$ by Lemma 3.5, and then the assertion $(*)$ in conjunction with the compactness of $C$ and the regularity of $E$ implies that $(T-i)(Y) \cap C \neq \varnothing$, that is, there exists a $\hat{y} \in Y$ such that $T(\hat{y}) \cap(\hat{y}+C) \neq \varnothing$. In case that $(3.6 .3)^{\prime}$ holds, since $(T-i)(Y)$ is compact by Lemma 2.1 and since $C$ is closed, the conclusion follows as the previous case. Finally, assume that $(3.6 .3)^{\prime \prime}$ holds. By $(*)$, for every convex open neighborhood $V$ of 0 , there are $y_{V}$ and $z_{V}$ in $Y$ such that $z_{V} \in T\left(y_{V}\right)$ and $z_{V}-y_{V} \in V$. Since $\overline{T(Y)}$ is compact, we may assume that $z_{V} \rightarrow \hat{y}$ for some $\hat{y} \in \overline{T(Y)}$. Then we also have that $y_{V} \rightarrow \hat{y}$. The closedness of $T$ implies that $\hat{y} \in T(\hat{y})$. This completes the proof.

The above corollary extends Park [11, Theorem 3], which in turn is a generalization to Lassonde [9, Theorem 1.6 and Corollary 1.18].

We now turn to investigate the fixed point problem on uniform spaces. At first we apply Theorem 3.2 to establish a useful lemma.

Lemma 3.7. Let $X$ be any nonempty set, $(Y, D ; \Gamma)$ be a $G$-convex space whose topology is induced by a uniformity $U$. Suppose $s: X \rightarrow D$ and $T \in s-\operatorname{KKM}(X, Y, Y)$ satisfy that

(3.7.1) $T$ is compact; and

(3.7.2) $\overline{T(Y)} \subseteq s(X)$.

If $V \in \mathcal{U}$ is symmetric and satisfies that $V[y]$ is $\Gamma$-convex for any $y \in Y$, then there is $y_{V} \in Y$ such that

$$
V\left[y_{V}\right] \cap T\left(y_{V}\right) \neq \varnothing .
$$


Proof. Define $H: Y \rightarrow 2^{Y}$ to be $H(y)=V[y]$ for any $y \in Y$. By symmetry of $V$ it is easy to see that $H^{-}(z)=V[z]$ for any $z \in Y$, and so $H^{-}(z)$ is $\Gamma$-convex. Also, it follows from condition (3.6.2) that for any $z \in \overline{T(Y)}$, there is $x_{0} \in s(X)$ such that $z=s\left(x_{0}\right)$. Then in view of $\left(s\left(x_{0}\right), s\left(x_{0}\right)\right) \in V$ we see that $z=s\left(x_{0}\right) \in V\left[s\left(x_{0}\right)\right]=H\left(s\left(x_{0}\right)\right)$, and hence $z \in \bigcup_{x \in X} H(s(x))$, that is $\overline{T(Y)} \subseteq \bigcup_{x \in X} H(s(x))$. Finally, noting $H$ is open-valued and putting $W: D \rightarrow 2^{Y}$ to be $W(y)=H(y)$ for any $y \in D$, we see that all the requirements of Theorem 3.2 are satisfied. Thus there is $y_{V} \in Y$ such that $H\left(y_{V}\right) \cap T\left(y_{V}\right) \neq \varnothing$, that is $V\left[y_{V}\right] \cap T\left(y_{V}\right) \neq \varnothing$.

Definition 3.8 [14]. A $G$-convex space $(X, D ; \Gamma)$ is said to be a locally $G$-convex uniform space if the topology of $X$ is induced by a uniformity $U$ which has a base $\mathcal{N}$ consisting of symmetric entourages such that for any $V \in \mathcal{N}$ and $x \in X, V[x]$ is $\Gamma$-convex.

Recall that the concepts of l.c. space and l.c. metric space in Horvath [7]. If $D=X$ and $\Gamma_{x}=\{x\}$ for any $x \in X$, then it is obvious that both of them are examples of locally $G$-convex uniform space.

Theorem 3.9. Let $X$ be any nonempty set, $(Y, D ; \Gamma)$ a locally $G$-convex space. Suppose $s$ : $X \rightarrow D$ and $T \in s-\operatorname{KKM}(X, Y, Y)$ satisfy that

(3.9.1) $T$ is compact and closed;

(3.9.2) $\overline{T(Y)} \subseteq s(X)$.

Then $T$ has a fixed point.

Proof. By Lemma 3.7, for any $V \in \mathcal{N}$ there is $y_{V} \in Y$ such that $V\left[y_{V}\right] \cap T\left(y_{V}\right) \neq \varnothing$. Choose $z_{V} \in V\left[y_{V}\right] \cap T\left(y_{V}\right)$. Then $\left(y_{V}, z_{V}\right) \in V \cap \operatorname{Gr}(T)$. Since $T$ is compact, we may assume that $\left\{z_{V}\right\}_{V \in \mathcal{N}}$ converges to $z_{0}$. For any $W \in \mathcal{N}$, choose $U \in \mathcal{N}$ such that $U \circ U \subseteq W$. Since $\left\{z_{V}\right\}_{V \in \mathcal{N}}$ converges to $z_{0}$, there is $V_{0} \in \mathcal{N}$ such that $V_{0} \subseteq U$ and

$$
z_{V} \in U\left[z_{0}\right], \quad \forall V \in \mathcal{N} \text { with } V \subseteq V_{0},
$$

that is,

$$
\left(z_{V}, z_{0}\right) \in U, \quad \forall V \in \mathcal{N} \text { with } V \subseteq V_{0}
$$

Thus, for $V \in \mathcal{N}$ with $V \subseteq V_{0}$, it follows from

$$
\left(y_{V}, z_{V}\right) \in V \subseteq U, \quad\left(z_{V}, z_{0}\right) \in U
$$

that $\left(y_{V}, z_{0}\right) \in U \circ U \subseteq W$. Hence $y_{V} \in W\left[z_{0}\right]$. This shows that $\left\{y_{V}\right\}_{V \in \mathcal{N}}$ converges to $z_{0}$. Since $T$ is closed, we conclude that $z_{0} \in T\left(z_{0}\right)$, completing the proof.

For a topological space $X$ and locally $G$-convex uniform space $(Y, \Gamma)$, define

$$
\begin{aligned}
T \in \mathscr{K}(X, Y) \Longleftrightarrow & T: X \longrightarrow Y \text { is a Kakutani map, that is, } \\
& T \text { is u.s.c. with nonempty compact } \Gamma \text {-convex values. }
\end{aligned}
$$

$\mathscr{K}_{c}(X, Y)$ denotes the set of finite composites of multimaps in $\mathscr{K}$ of which ranges are contained in locally $G$-convex uniform spaces $\left(Y_{i}, \Gamma_{i}\right)(i=0, \ldots, n)$ for some $n$. 
Lemma 3.10 (Watson [14]). Let $(X, \Gamma)$ be a compact locally $G$-convex uniform space. Then any u.s.c. $T: X \multimap X$ with closed $\Gamma$-convex values has a fixed point.

By the above lemma, we see that, in the setting of locally $G$-convex uniform spaces, the class $\mathscr{K}$ is an example of the Park's class U. Therefore, for any locally $G$-convex uniform space $(X, \Gamma), \mathscr{K}_{c}(X, X) \subseteq \mathrm{KKM}(X, X)$, and so we have the following theorem.

Theorem 3.11. Suppose $(X, \Gamma)$ is a locally $G$-convex uniform space. If $T \in \mathscr{K}_{c}(X, X)$ is compact, then it has a fixed point.

Proof. Since $X$ is regular by Kelley [8, Corollary 6.17 on page 188 ] and $T \in \mathcal{K}_{c}(X, X)$, it is u.s.c. and compact-valued, and so it is closed. Now due to that $\mathscr{K}_{c}(X, X) \subseteq \operatorname{KKM}(X, X)$, we have $T \in \operatorname{KKM}(X, X)$. Since $T$ is compact and closed, it follows from Theorem 3.9 that $T$ has a fixed point.

Since any metric space is regular, we infer that for any l.c. metric space $(X, d)$ satisfying that $\Gamma_{x}=\{x\}$, if $T \in \mathscr{K}_{c}(X, X)$ is compact, then $T$ has a fixed point. This generalizes the famous Fan-Glicksberg fixed point theorem [5].

\section{References}

[1] F. E. Browder, The fixed point theory of multi-valued mappings in topological vector spaces, Mathematische Annalen 177 (1968), 283-301.

[2] _ Coincidence theorems, minimax theorems, and variational inequalities, Conference in Modern Analysis and Probability (New Haven, Conn, 1982), Contemp. Math., vol. 26, American Mathematical Society, Rhode Island, 1984, pp. 67-80.

[3] T.-H. Chang, Y.-Y. Huang, J.-C. Jeng, and K.-H. Kuo, On S-KKM property and related topics, Journal of Mathematical Analysis and Applications 229 (1999), no. 1, 212-227.

[4] T.-H. Chang and C.-L. Yen, KKM property and fixed point theorems, Journal of Mathematical Analysis and Applications 203 (1996), no. 1, 224-235.

[5] K. Fan, A generalization of Tychonoff's fixed point theorem, Mathematische Annalen 142 (1960/1961), 305-310.

[6] A. Granas and F. C. Liu, Coincidences for set-valued maps and minimax inequalities, Journal de Mathématiques Pures et Appliquées. Neuvième Série(9) 65 (1986), no. 2, 119-148.

[7] C. D. Horvath, Contractibility and generalized convexity, Journal of Mathematical Analysis and Applications 156 (1991), no. 2, 341-357.

[8] J. L. Kelley, General Topology, D. Van Nostrand, Toronto, 1955.

[9] M. Lassonde, On the use of KKM multifunctions in fixed point theory and related topics, Journal of Mathematical Analysis and Applications 97 (1983), no. 1, 151-201.

[10] L.-J. Lin and S. Park, On some generalized quasi-equilibrium problems, Journal of Mathematical Analysis and Applications 224 (1998), no. 2, 167-181.

[11] S. Park, Foundations of the KKM theory via coincidences of composites of upper semicontinuous maps, Journal of the Korean Mathematical Society 31 (1994), no. 3, 493-519.

[12] S. Park and H. Kim, Coincidence theorems for admissible multifunctions on generalized convex spaces, Journal of Mathematical Analysis and Applications 197 (1996), no. 1, 173-187. 
[13] S. Park and H. Kim, Foundations of the KKM theory on generalized convex spaces, Journal of Mathematical Analysis and Applications 209 (1997), no. 2, 551-571.

[14] P. J. Watson, Coincidences and fixed points in locally G-convex spaces, Bulletin of the Australian Mathematical Society 59 (1999), no. 2, 297-304.

Tian-Yuan Kuo: Fooyin University, 151 Chin-Hsueh Rd., Ta-Liao Hsiang,

Kaohsiung Hsien 831, Taiwan

E-mail address: sc038@mail.fy.edu.tw

Young-Ye Huang: Center for General Education, Southern Taiwan University of Technology, 1 Nan-Tai St. Yung-Kang City, Tainan Hsien 710, Taiwan

E-mail address: yueh@mail.stut.edu.tw

Jyh-Chung Jeng: Nan-Jeon Institute of Technology, Yen-Shui, Tainan Hsien 737, Taiwan

E-mail address: jhychung@pchome.com.tw

Chen-Yuh Shih: Department of Mathmatics, Cheng Kung University, Tainan 701, Taiwan

E-mail address: cyshih@math.ncku.edu.tw 